\title{
Infrared thermography of human face for monitoring thermoregulation performance and estimating personal thermal comfort
}

\author{
Ali Ghahramani ${ }^{\text {a }}$, Guillermo Castro ${ }^{\mathrm{b}}$, Burcin Becerik-Gerber ${ }^{\mathrm{c}}$, Xinran $\mathrm{Yu}^{\mathrm{d}}$ \\ ${ }^{a}$ PhD Student, Sonny Astani Dept. of Civil and Environmental Engineering, Viterbi School of \\ Engineering, Univ. of Southern California, KAP 217, 3620 South Vermont Ave., Los Angeles, CA \\ 90089-2531. E-mail: aghahram@usc.edu \\ ${ }^{\mathrm{b}}$ Research Assistant, Sonny Astani Dept. of Civil and Environmental Engineering, Viterbi School of \\ Engineering, Univ. of Southern California, KAP 217, 3620 South Vermont Ave., Los Angeles, CA \\ 90089-2531. E-mail: guille131g@gmail.com \\ ${ }^{\mathrm{c}}$ Associate Professor, Sonny Astani Dept. of Civil and Environmental Engineering, Viterbi School of \\ Engineering, Univ. of Southern California, KAP 224C, 3620 South Vermont Ave., Los Angeles, CA \\ 90089-2531. E-mail: becerik@usc.edu, Tel: +1 2137404383 (Corresponding Author) \\ ${ }^{\mathrm{d}}$ Graduate Student, Sonny Astani Dept. of Civil and Environmental Engineering, Viterbi School of \\ Engineering, Univ. of Southern California, KAP 217, 3620 South Vermont Ave., Los Angeles, CA \\ 90089-2531. E-mail: xinranyu@usc.edu
}

\begin{abstract}
The common practice of defining operational settings for Heating, Ventilation and Air Conditioning (HVAC) systems in buildings is to use fixed set points, which assume occupants have same and static comfort requirements. However, thermal comfort varies from person to person and also changes due to climatic variations or acclimation, making it dynamic. In addition, thermal comfort in transient conditions are different from the steady state conditions, which makes the prediction of thermal comfort more difficult. Thus, thermal comfort has to be monitored over time. In this paper, we present a novel infrared thermography based technique to monitor an individual's thermoregulation performance and thermal comfort levels by measuring the skin temperature on several points on human face, which has a high density of blood vessels and is not usually covered by clothing. Unlike other methods, our method requires no continuous user input or interaction. Our results demonstrate that the monitored facial points behave differently under the heat and cold stresses and it can be explained based on the underlying vascular territories. We define two heuristics to describe the thermoneutral zone based on the observed behaviors and estimate thermal comfort for individuals with $95 \%$ confidence level. Considerable variations are observed in the thermoregulation performance and uncomfortably cool conditions metrics between the males and females. Females' thermoregulation system responses are less sensitive to the perception of warm conditions. However, similar behaviors are observed for uncomfortably cool conditions across genders.
\end{abstract}

Keywords: Thermoregulation system; Thermal comfort; Infrared thermography; Energy efficiency; Sensing; Physiological measurements

\section{INTRODUCTION}

Commercial and residential buildings account for $30 \%$ of the total energy consumption [1] and $30 \%$ of the greenhouse gas emissions in the world [2]. Heating, Ventilation and Air Conditioning (HVAC) systems, which provide thermal comfort and air quality in buildings, account for the largest share of the energy usage and gas emissions (about $50 \%$ of the building energy consumption in the developed 
countries [3]). HVAC systems are usually operated with fixed setpoints based on the recommendations provided by the standards (e.g., ASHRAE Standard 55 [4], ASHRAE Standard 62.1 [5]). These standards for thermal comfort offer models that use few selected parameters (e.g., indoor air temperature, air humidity, clothing, activity) to estimate thermal comfort. However, some of the static and dynamic factors that are influential on thermal comfort are not considered in these models. Factors that are constant over time are the static factors, such as a person's race and gender. Factors that impact an individual's thermal comfort over time are the dynamic factors, such as age, acclimation, prior heat or cold stress, and food intake [6-8]. Even though it is difficult to assess human comfort in real-time due to these factors, building HVAC systems energy consumption can still be optimized while occupants thermal comfort is wellmaintained [9] as humans perceive comfort in a range of environmental thermal conditions [10-12]. It is worth mentioning that small thermal comfort related adjustments (e.g., adjusting the temperature set point by $1^{\circ} \mathrm{C}$ ) have considerable impacts on the overall building energy consumption $[13,14]$.

Thermal comfort is defined as the condition of mind [5] and thus it can only be captured by surveying individuals about their comfort. Due to the fact it is not practical for building occupants to communicate their thermal comfort levels via a questionnaire on a continuous basis, researchers have proposed different techniques to correlate environmental or physiological measurements with thermal comfort (usually reported by the occupants via a questionnaire) and use these environmental or physiological measurements for predicting individuals' thermal comfort. Although environmental measurement based techniques have shown promising results to capture thermal comfort in short periods of time, the accuracy of these methods may decay due to environment related logistics (e.g., the sensing devices or occupants' locations may change in time). To address the challenge of occupant-environment logistics that are dynamic, the techniques based on physiological measurements (e.g., the techniques that use heart rate, skin temperature, and core body temperature $[8,15-17])$ use the correlated measurements directly from an individual for predicting thermal comfort. If there is no evidence of thermal discomfort, these approaches reject the hypothesis that the individual is uncomfortable. The drawbacks of these techniques is that they require the sensors to be attached to the human body, making them invasive.

In this paper, we present a novel infrared thermography sensing technique to monitor an individual's thermoregulation performance, as well as thermal comfort in a non-invasive manner. Our proposed technique uses the fact that humans, similar to other endothermic animals, maintain optimal physical operations and potentially perceive comfort when they are in a thermoneutral zone [18] -- the range of operating temperatures where the body temperature is maintained without thermoregulatory changes (e.g., shivering or sweating) [19]. We indirectly measure the variations of blood circulation in the cutaneous vessels on human face and map these variations first to the thermoregulation system performance and then to thermal comfort. The paper is structured as follows. Section 2 provides a literature review of the recent studies on personal comfort learning methods that use physiological measurements. Section 3 focuses on the thermoregulation system and provides a background on how skin blood flow varies in response to thermal stresses, as well as a review of skin blood variation detection methods. In Section 4, we explain our data collection methodology. Section 5 presents our methodology for quantifying the performance of the thermoregulation system and defining the heuristics for thermal comfort, as well as the results and modeling accuracy in a sequential manner. Section 6 provides a discussion on the limitations and potential future research. Finally, Section 7 summarizes the findings and concludes the paper. 


\section{PHYSIOLOGICAL SENSING OF THERMAL COMFORT}

The recent advancements in the human computer interaction fields and specifically the participatory sensing approaches have led to the development of the web interfaces or apps that communicate with building management systems to make the indoor thermal environments comfortable based on the thermal comfort feedback received from the building occupants. The drawback with these methods is that they require continuous and frequent user feedback. To capture the dynamic variations in the personal thermal comfort and reduce the need for occupants to continuously provide feedback about their thermal comfort, researchers have explored several techniques, which predominantly differ based on the data acquisition methods. Accordingly these methods can be categorized into two groups (1) environmental measurement based techniques; and (2) physiological measurement based techniques [20]. Environmental measurement based techniques use supervised learning methods to correlate the environmental conditions with occupant feedback and estimate thermal comfort in the absence of the feedback. There are challenges that make the applications of these techniques difficult to generalize, such as the relationship between the location of the sensing devices and occupants in the built environment, distribution of the air temperature gradient, and other properties of the environment that make the learning difficult. A detailed review of these methods could be found in [12].

Physiological measurement based techniques are built on the principle that physiological responses can be correlated with thermal discomfort $[15,16,21]$. Monitoring of the correlated measurements enables detection of the conditions that an individual is likely to become uncomfortable. If there is no evidence of thermal discomfort, these approaches reject the hypothesis that the individual is in an uncomfortable condition. Consequently, physiological measurement based techniques are potentially capable of addressing the issues related to the environmental measurement based techniques by directly sensing the occupants. The authors in [22] developed an exergy based approach that relates an individual's body exergy consumption rate with their assessed thermal sensations. Their results suggested that minimum body exergy consumption rate is associated with the sensation close to the thermal neutrality. In addition, the authors found considering both the convective and radiative heat exchange between a human body and the environment, indoor operative temperature is an appropriate measure for estimating the body exergy consumption rate. A multiple regression model that takes the mean skin temperature and its time differential as input and predicts transient thermal sensations was introduced in [16]. The results showed a strong correlation (a correlation coefficient of 0.839) for predicting the sensations using the proposed technique. A data driven modeling technique that combines personalized coefficients with a general model of human body heat balance was introduced in [23]. The coefficients were estimated by minimizing a least square error function of the coefficients based on the comfort votes collected over time. The authors argued that the data communicated by the occupants on a daily basis account for the adaptive changes in the model. The authors in [24] used individuals' heart rate and electroencephalograph (EEG) measurements as a representation of thermal comfort and investigated the relationship between the measurements and environmental temperature variations. They found heart rate measurements are more correlated (with statistical significance) with thermal comfort votes collected during the data collection period. In [25], the authors investigated the applicability of using individuals' mean skin temperature as a representation of thermal comfort. Various combinations of average sensor measurements on 26 points on the human body were explored and the average of 10 points were chosen

as the most accurate measure (R-squared measure of above 0.9). The authors in [26] developed a wrist band that monitors skin temperatures on a wrist at several points, such as the radial artery and ulnar artery 
regions, and upper wrist) and from fingertips and collected data from eight subjects under different thermal conditions. Accordingly, a thermal sensation estimation model based on the mean skin temperature, temperature gradient, time differential of the temperatures, and average power of frequency band were developed. The validation results showed that a personalized thermal sensation estimation model based on three wrist skin temperatures had the mean RMSE of $1.06 \pm 0.29$, with a correlation coefficient of 0.89 . The authors in [27] developed an estimation algorithm for individual's thermal sensations based on the peripheral skin temperature measurements. They used modified environmental temperatures to explore the relationship between peripheral skin temperature and thermal sensation votes. Their results demonstrated a mean square error of below 1 for estimating thermal sensations. In another study [28], the authors used several points on human body as measurement points and studied the relationship of the measurements with the overall thermal sensation. They found that the finger skin temperature and finger-forearm temperature gradient have a high correlation $(r=0.78$ and 0.80 , respectively) with the overall thermal sensation.

These models require the building occupants to continuously train the system for learning thermal comfort levels of individuals. In addition, the sensing devices (e.g., temperature, heart rate, and EEG sensors), used to collect data to build these models, have to be attached to the human body, making them invasive. In this paper, we introduce a novel sensing technique that does not require the sensors to be attached to a human body (as we use human body infrared radiations as measurements) to capture thermoregulation performance and thermal comfort.

\section{BACKGROUND ON THERMOREGULATION SYSTEM}

\subsection{Skin blood flow variations in response to thermal stresses}

Thermoregulation system maintains temperature homeostasis, which is the process of regulating internal body variables to keep the core body temperature within the range of approximately between $36^{\circ} \mathrm{C}$ and $38^{\circ} \mathrm{C}$. Temperature homeostasis allows for optimal operations of the internal organs while maintaining heat equilibrium with the environment. The temperatures that are thermally comfortable are a subset of temperatures when the body is in the thermoneutral zone [29]. When there is thermal stress (i.e., heat and cold) caused by external factors (e.g., increased temperature or humidity) or internal factors (e.g., food intake), human body responds and the thermoregulation system adjusts heat dissipation to the external environment by modifying the blood flow via cutaneous arterioles and veins, causing sweating or shivering [30]. Accordingly, resting skin blood flow in the arterioles in normothermic conditions is approximately $250 \mathrm{~mL} / \mathrm{min}$ (about $5 \%$ of the cardiac output [31]), which results in a heat dissipation of about 80 to $90 \mathrm{kcal} / \mathrm{h}$ ( $\sim$ the level of resting metabolic heat production) [32]. In response to the heat stress, thermoregulatory vasodilation can increase skin blood flow up to 6 to $8 \mathrm{~L} / \mathrm{min}$ [33] and utilize up to $60 \%$ of cardiac output [31]. In response to the cold stress, thermoregulatory vasoconstriction can limit the skin blood flow to approach zero. The dual sympathetic neural control mechanisms are performed via two populations of the sympathetic nerves. While non-glabrous skin is covered with both vasoconstrictor and vasodilator nerves, glabrous skin (e.g., skin on palms, soles and lips) are innervated only by sympathetic vasoconstrictor nerves [34]. Glabrous skin has a rich arteriovenous anastomoses, which are thick, low resistance conduits that allow high flow rates directly from arterioles to venules and are innervated by sympathetic vasoconstrictor nerves. Non-glabrous skin does have a very few arteriovenous anastomoses. This implies that the regions without the vasodilation, such as wrists, do not provide appropriate representations of thermoregulation performance. 
The vasoconstrictor system is tonically active in thermoneutral environments [35]. Slight changes in the skin blood flow can result in relatively large changes in heat transfer to the environment (an increase in skin blood flow by $8 \%$ over the entire body results in doubling the heat transfer to the environment) [32]. Solely through the changes in the cutaneous vasomotor tone, temperature homeostasis can be achieved. Sympathetic vasodilator system is not tonically active in normothermia and is only activated when internal temperature increases (e.g., during exercise or heat exposure) [36]. The vasoconstrictor system immediately activates and reduces blood flow during cold stress. After removal of the cold stress, the skin blood flow immediately returns to the normothermia conditions. In addition, vasoconstrictor system can help dissipating in heat stress via relaxing the blood vessels to increase the blood flow. Vasodilation and sweating begin when the internal body temperature approaches a temperature threshold. The distribution of cutaneous vessels is not uniform across the body (Figure 1).

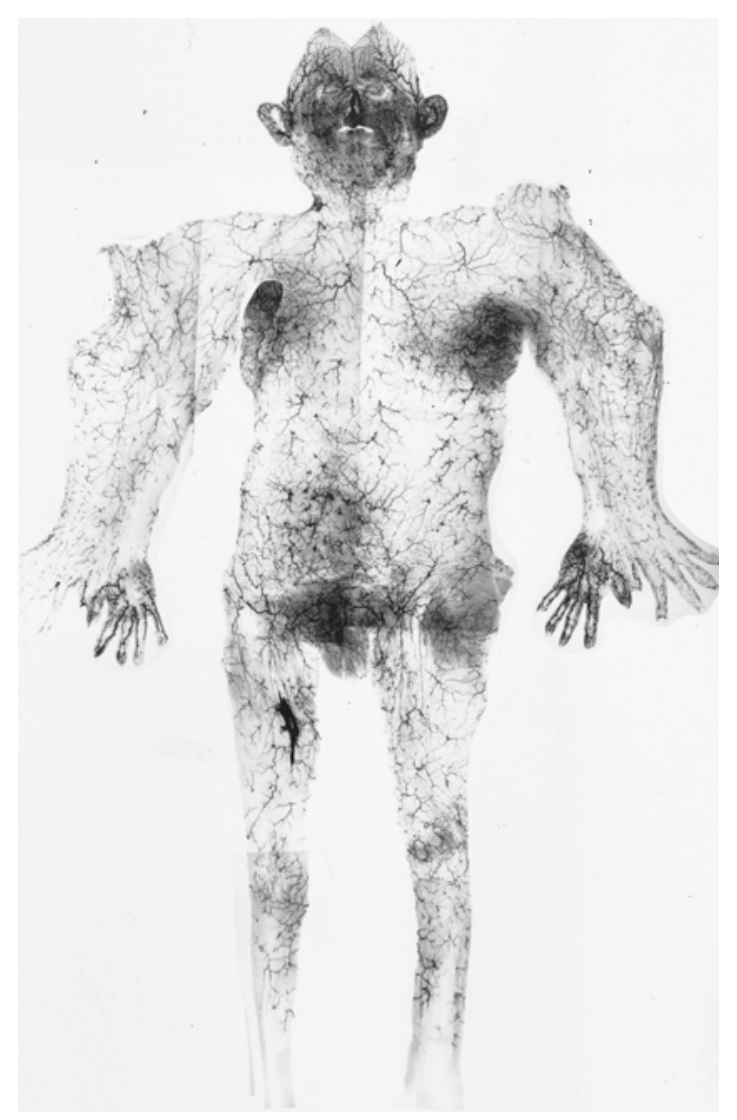

Figure 1. Cutaneous arteries of a male human [30].

\subsection{Detection methods for skin blood flow variations}

There are several methods for measuring skin blood flow, including venous occlusion plethysmography, laser Doppler, ultrasound, thermostrom, photoelectric plethysmography, impedance, and radioactive isotopes $[37,38]$. Venous occlusion plethysmography (VOP) measures the blood flow by measuring the change in the volume of an organ or limb, usually the forearm [39]. VOP uses a cuff to stop venous drainage from leaving the limb and only allows the blood to enter through arteries, causing a linear increase in volume, which is proportional to the arterial blood inflow. However, because the VOP renders 
hand ischemia, it cannot gather blood flow data for more than 13 minutes, and it cannot be used during exercise or any other type of movement. Doppler ultrasound uses the Doppler technique to measure the speed of blood flow velocity with ultrasound and finds conduit vessel diameters to determine blood flow [37]. The ultrasound head must always be perpendicular to the artery and any movement results in a large error. There are a few laser Doppler techniques, which are the most common ways to measure the blood flow. These techniques use laser frequency instead of ultrasound. Because of the many variations (e.g., heart rate, respiration rate, etc.) in the blood flow, these techniques must measure for a minimum of $20 \mathrm{~s}$ to ascertain an average rate. The most accurate laser Doppler technique is the large beam laser [37]. Disadvantages of the laser Doppler include: measurements get thrown off by pigment variations, movement and high temperatures alter blood flow so subjects must wait up to 30 min after walking in, pressure cannot be applied to the skin, light level of activity must be kept low, and any type of movement must be avoided while being screened. The thermistor method uses a heated thermistor pair that changes electrical resistance with temperature. A thermistor is placed on the skin, and the skin blood flow heats up the thermistor, which increases the resistance, increasing the current needed to heat the other thermistor. The measured current is proportional to the blood flow. This method is advantageous because it samples over a large area and reduces motion artifacts. Hertzman photoelectric plethysmography (HPP) uses an infrared light source at 45 degrees to the skin and a photocell perpendicular to the skin. The transmission of infrared light measures the blood flow. Motion results in large errors, and HPP can only measure relative blood flows [37]. However, it is inexpensive and can be used for both skin and organs. Impedance uses four electrodes to measure changes in tissue conductivity to measure blood flow. It does not differentiate between the skin and limb blood flow and is affected by body hydration levels, race, age, and limb fat content. Radioactive isotopes can also be used to measure skin blood flow, however it is a very expensive method and ions can be absorbed by body fat.

Even though the above mentioned methods measure blood flow directly, they have several limitations that prevent their use in learning thermoregulation system performance and/or estimating personal thermal comfort. These limitations include: the need to be attached to an organ or limb, need to avoid any movement by subjects as motion results in large errors [40], need to use the device with a certain angle or position, need for light level of activity to be maintained, getting affected by body hydration levels, race, age, and limb fat content. In this study, as an alternative to the direct skin blood flow measurements, we used skin infrared radiations (i.e., skin temperature) as an indirect measurement of skin blood flow. As explained below, our data acquisition system, using an infrared thermography sensing technique, allows for real-time non-invasive measurements of skin temperatures, which we use for estimating thermal comfort.

\section{DATA COLLECTION METHODS AND PROCEDURES}

For our explorations, we designed an experiment that included 15 participants (10 males and 5 females; 2 African American, 4 Hispanic, 4 Asian, and 5 White participants) to monitor the human thermoregulation performance during hot and cold thermal stresses while collecting subjective thermal comfort votes. Thermal comfort votes are the conscious perceptions of an individual to a thermal environment, whereas the thermoregulation system regulates the unconscious responses of an individual to the thermal environment. The data collection for our experiments was completed in an office space in a building on a university campus in California. The climate of the area is defined as a dry-summer subtropical climate (aka, the Mediterranean climate) based on the Köppen climate classification [41]. For such climates, the average temperature in the warm months is above $10^{\circ} \mathrm{C}$ and in the cold months is between -3 and $18^{\circ} \mathrm{C}$. 
We performed the data collection between July 2015 and October 2015. The experiment duration for each participant was four days with at least two hours a day. During the first three days (comfortable days), comfortable conditions based on the participants' preferences were provided. In the fourth day, the HVAC system was deliberately set to extreme temperatures (extreme day) to expose the participants to different thermal stimuli. All of the participants started the extreme day at a comfortable condition (a temperature value that differed between the participants based on their personal preferences). They were randomly assigned comfortable to high to low (i.e., comfortable condition to $29^{\circ} \mathrm{C}$ to $18^{\circ} \mathrm{C}$ ) or comfortable to low to high (i.e., comfortable condition to $18^{\circ} \mathrm{C}$ to $29^{\circ} \mathrm{C}$ ) temperature settings. We randomly assigned the participants to eliminate the bias that might have occurred due to a specific heat gradient condition.

To understand human thermoregulation responses to the thermal stimuli, we used a non-invasive method for indirectly measuring the skin blood flow by infrared thermography using infrared sensors. As explained in Section 3, the distribution of the cutaneous vessels is not uniform across a human body. On areas around face, the density of the vessels is considerably higher, which enables higher blood circulation. In addition, human face is usually not covered with clothing in commercial buildings. Thus, we used facial skin temperature (Figure 2a) as a measure of skin blood flow via an eyeglass frame with infrared sensors installed on it (Figure $2 b$ ) to characterize the thermoregulation responses of human body during heat and cold stresses. Figure 3 illustrates a male participant wearing the glass frame with the attached infrared sensing system.

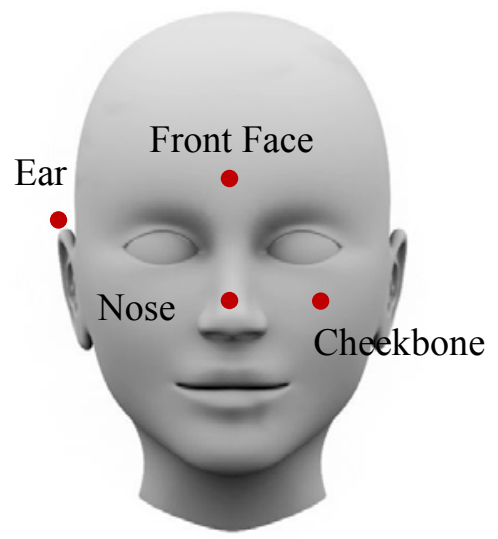

(a)

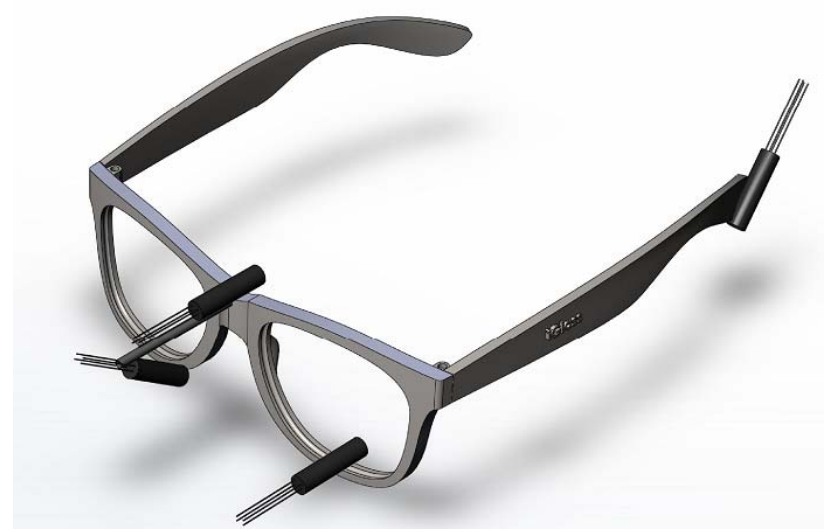

(b)

Figure 2. (a) Approximate infrared sensing locations on face and (b) 3D view of sensing device with four infrared sensors 


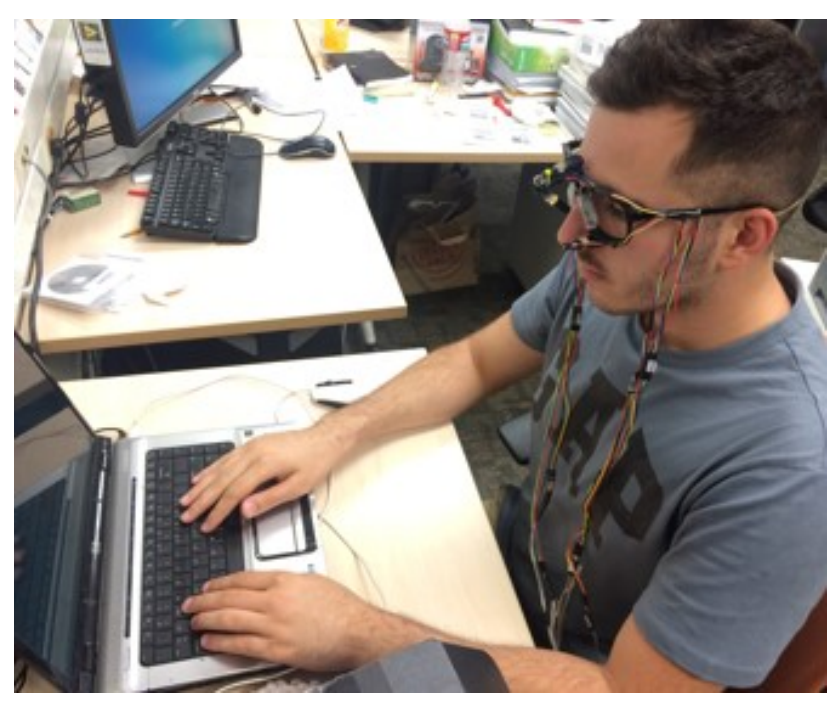

Figure 3. A male participant wearing the glass frame with infrared sensing system.

Each participant was given an ID number and asked to communicate his/her votes with that ID number, using a user interface (Figure 4) with 7 scales to cover different thermal comfort levels [42]. The thermal comfort scale allowed a selection from the following options: much too warm, uncomfortably warm, comfortably warm, comfortable, comfortably cool, uncomfortably cool and much too cool. We grouped comfortably warm, comfortable, and comfortably cool votes as the comfortable condition, and much too warm and uncomfortably warm votes as the uncomfortably warm condition, and uncomfortably cool and much too cool votes as the uncomfortably cool condition for our data analysis as the primary investigation was about comfortable versus uncomfortable conditions. The participants were asked to communicate their votes at least 10 votes per day with at least a 15 -minute interval between each vote. The participants provided their feedback while performing regular office activities (e.g., reading, writing and working on a computer).

Please Choose Your ID 101 v

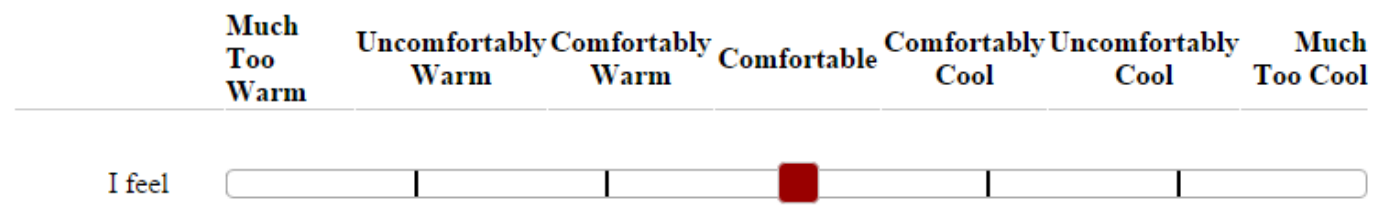

Figure 4. User interface for collecting personal thermal comfort votes

Two electrical heaters and a dedicated air conditioning system (controlled with a thermostat) were the heating and cooling sources in the office space, respectively. These systems provide the heat and cold stimuli through mainly adjusting air temperature, and consequently we took air temperature as a representation of thermal stimuli. The relative humidity during our experiments was between $45 \%$ and $55 \%$. It is worth mentioning that the participants were seated in a way that they did not experience direct flow of heat or cold. The plan of the office space is illustrated in Figure 5. 


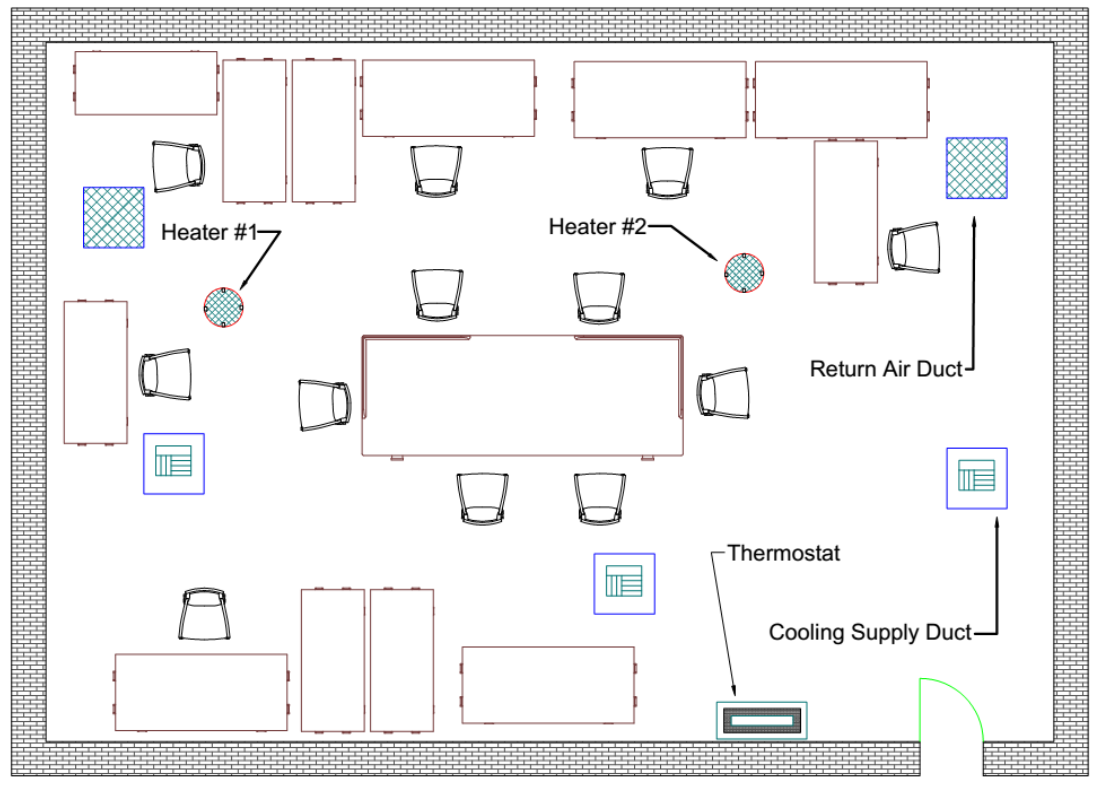

Figure 5. Office space floor plan

We also collected the operative temperatures around each participant as the signature of the thermal stimuli in the environment. The temperature/humidity sensor used in the experiments was Aosong AM2302 temperature/humidity sensor, which has a resolution of $0.1^{\circ} \mathrm{C}$ for temperature and $1 \%$ $\mathrm{RH}$ (Relative Humidity) for humidity and an accuracy of $\pm 0.5^{\circ} \mathrm{C}$ for temperature and $\pm 2 \% \mathrm{RH}$ for temperature. The sensors were placed in a closed proximity to the participants. We placed four sensors around the participants to make sure no local heating or cooling impact the results of the study. For the numerical analysis, we used the average temperature readings in the later sections. The infrared sensors were MLX90614, which has an accuracy of $\pm 0.1^{\circ} \mathrm{C}$ for temperature with the resolution of $0.01{ }^{\circ} \mathrm{C}$. The resolution and accuracy provided by the infrared sensor enabled accurate measurements of skin temperature. The infrared sensor was recommended by the producers to be used for medical services [43]. The infrared sensor calculates the average temperature of the surfaces in its field view and reports it as a scalar variable. It should be noted that there could be errors introduced by external sources (e.g., reflected radiation from surrounding, air temperature and air flow speed, etc.) that may impact the sensor performance. Accordingly, necessary precautions were taken during the data collection period. For example, the electrical heaters were located in positions that had minimal impacts on the measurements. Sensors were connected by multiple Arduino boards that were responsible to collect the data and store them in a database as time-series. The Arduino board was Arduino Uno, which has a $16 \mathrm{MHz}$ processor and a $32 \mathrm{kB}$ memory and the connections to the sensors and the computer, hosting the database, were wired.

\section{METHODS AND RESULTS}

By monitoring the thermoregulation performance, we aim to identify the thermoneutral zone and consequently predict the thermal comfort. Accordingly, we first explored if we can detect and monitor the body thermoregulation performance in response to the cold and heat stresses through skin infrared radiation (i.e., skin temperature) measurements. We then map cardiovascular territories into the 
thermoregulation performance and explored if we can estimate thermal comfort by monitoring the thermoregulatory performance.

\subsection{Correlation analysis between thermoregulatory performance and thermal stimuli measurements}

Figure 6 shows the data (cheekbone, ear, front face, nose, room temperatures, as well as average facial point temperature) of two participants over 4 days. The vertical lines (at the end of each day) demonstrate the end of the data acquisition for each day. Accordingly, there are three dashed lines, which represent the first three days, followed by the fourth day's data.

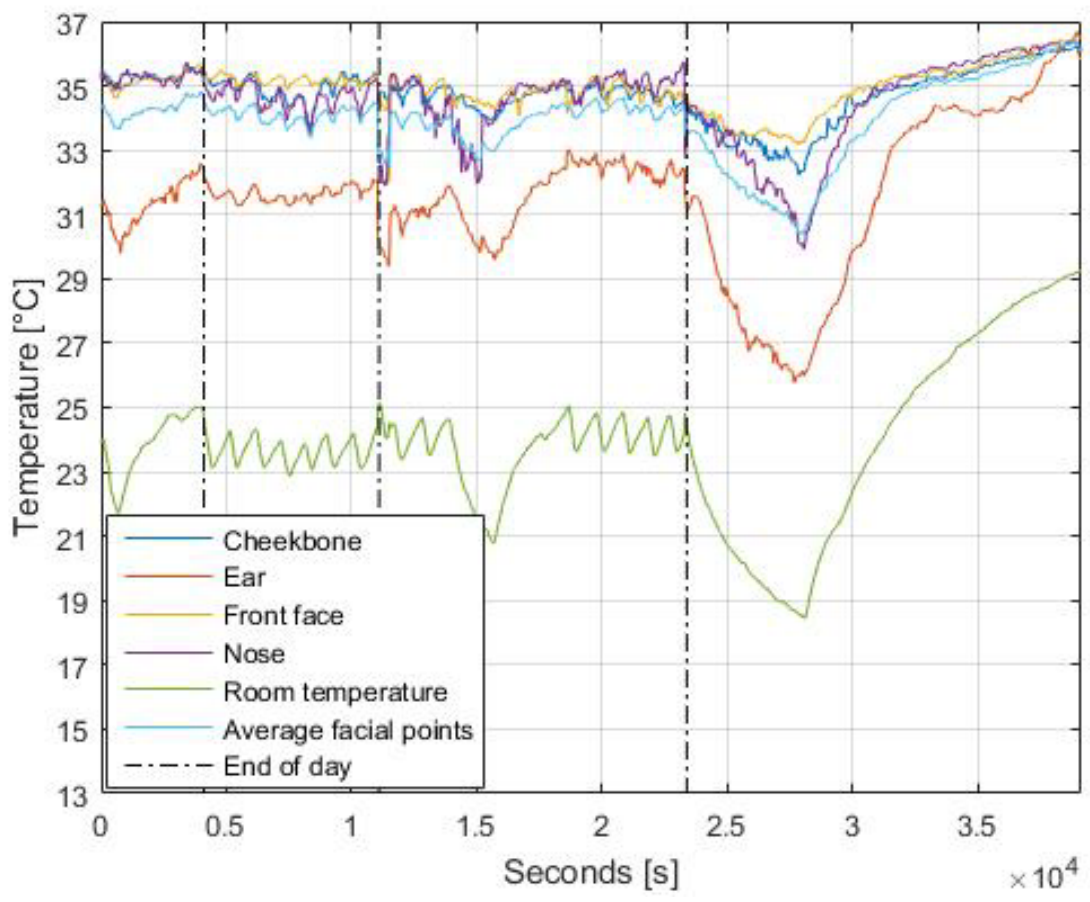




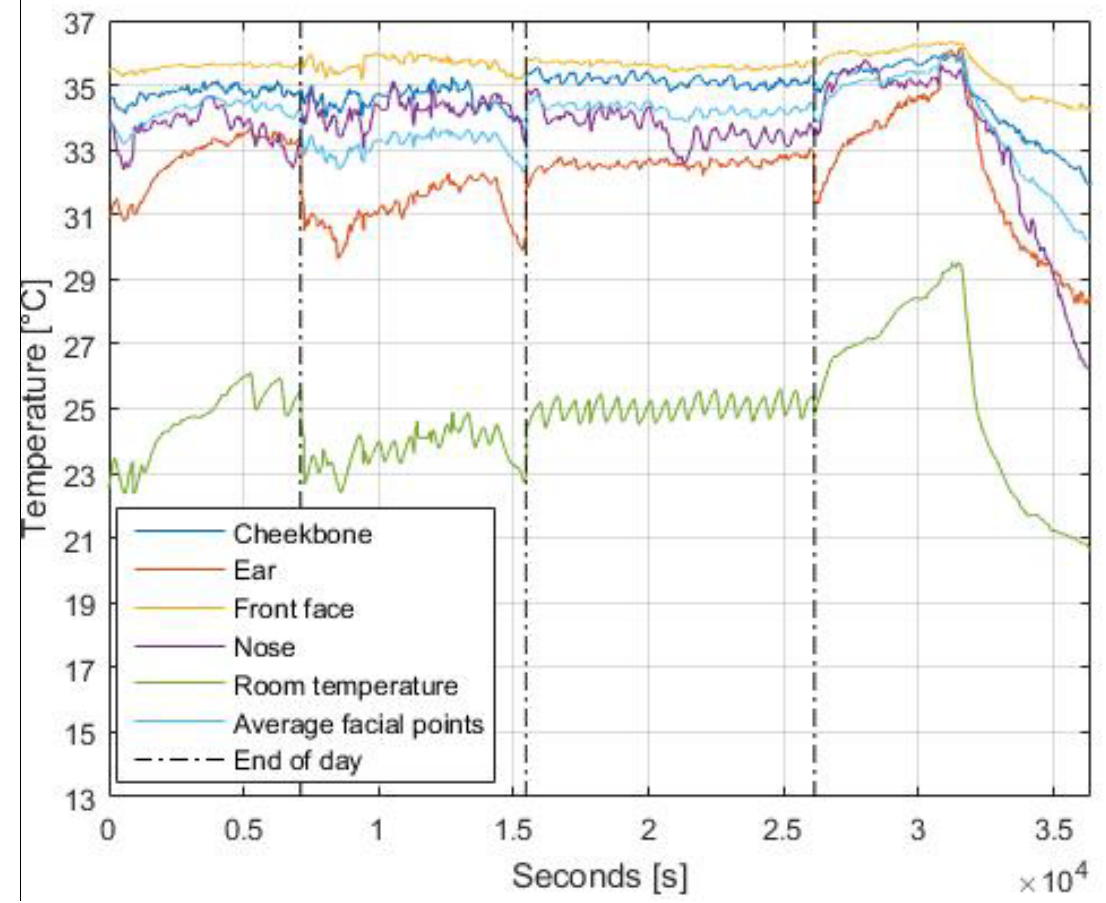

Figure 6. Facial point measurements over 4 days of the experiment for two participants

As it can be seen in Figure 6, the facial measurements and the room temperatures follow similar patterns. However, single measurements on face behave slightly different (mostly during extreme days). This is due to the fact that the room temperature is one of the influential factors on the thermoregulation performance. In addition, there is a small gap between the temperature measurements at the beginning of each day This is due to the fact that several hours passed between the different periods of data collection, resulting in different temperature measurements. In order to investigate if we can detect and monitor the body thermoregulation performance in response to cold and heat stresses, we calculated the correlation coefficients between the thermoregulatory performance (each measured facial point and average facial points) and thermal stimuli (room temperature). The correlation coefficient of two random variables (e.g., $\mathrm{A}$ and $\mathrm{B}$ ) is a measure of their linear dependence. The Pearson correlation coefficient used in for correlation analysis is defined as (Equation 1):

$\rho(\mathrm{A}, \mathrm{B})=\frac{\operatorname{cov}(\mathrm{A}, \mathrm{B})}{\sigma_{\mathrm{A}} \sigma_{\mathrm{B}}}$

Equation 1

Where $\sigma_{A}$ the standard deviation of $\mathrm{A}$ is, $\sigma_{B}$ is the standard deviation of $\mathrm{B}$, and $\operatorname{cov}(A, B)$ is the covariance of $\mathrm{A}$ and $\mathrm{B}$. The correlation matrix is symmetric $(\rho(A, B)=\rho(B, A))$. Table 1 summarizes the data for the correlation analysis.

Table 1. Correlation matrix between various points

\begin{tabular}{|c|c|c|c|c|c|c|}
\cline { 2 - 7 } \multicolumn{1}{c|}{} & Cheekbone & Ear & Front Face & Nose & $\begin{array}{c}\text { Average } \\
\text { Facial Points }\end{array}$ & $\begin{array}{c}\text { Room } \\
\text { Temperature }\end{array}$ \\
\hline Cheekbone & 1.00 & $0.66 \pm 0.27$ & $0.82 \pm 0.12$ & $0.76 \pm 0.15$ & $0.79 \pm 0.12$ & $0.88 \pm 0.10$ \\
\hline Ear & $0.66 \pm 0.27$ & 1.00 & $0.64 \pm 0.21$ & $0.60 \pm 0.20$ & $0.75 \pm 0.14$ & $0.86 \pm 0.11$ \\
\hline Front Face & $0.82 \pm 0.12$ & $0.64 \pm 0.21$ & 1.00 & $0.70 \pm 0.16$ & $0.83 \pm 0.09$ & $0.84 \pm 0.09$ \\
\hline
\end{tabular}




\begin{tabular}{|c|c|c|c|c|c|c|}
\hline Nose & $0.76 \pm 0.15$ & $.60 \pm 0.20$ & $0.70 \pm 0.16$ & 1.00 & $0.67 \pm 0.12$ & $0.87 \pm 0.08$ \\
\hline $\begin{array}{c}\text { Average } \\
\text { Facial Points }\end{array}$ & $0.79 \pm 0.12$ & $0.75 \pm 0.14$ & $0.83 \pm 0.09$ & $0.67 \pm 0.12$ & 1.00 & $0.85 \pm 0.07$ \\
\hline $\begin{array}{c}\text { Room } \\
\text { Temperature }\end{array}$ & $0.88 \pm 0.10$ & $0.86 \pm 0.11$ & $0.84 \pm 0.09$ & $0.87 \pm 0.08$ & $0.85 \pm 0.07$ & 1.00 \\
\hline
\end{tabular}

The ear measurements have small correlations with the cheekbone, front face and nose measurements. Both the front face and cheekbone measurements have relatively smaller correlations with the nose measurements although the cheekbone and nose sensing points had a fairly smaller distance compared to the cheekbone and front face sensing points. The room temperature has a high correlation with average measured facial points, however not as much with individual facial points, suggesting that each point behaves differently, while the average values follow room temperature (see also Figure 6). However, the front face and cheekbone have a very similar behavior (high correlation coefficient). Such behavior can be explained based on the cardiovascular territories underlying the skin. Based on the blood supply to the cutaneous vessels and the underlying deep tissues, a human body can be segregated into three-dimensional vascular territories [30,44]. The anatomic territories are supplied by a source (segmental or distributing) artery and accompanying veins that span between skin and bone. Figure 7 illustrates the vascular territories of all tissues between the skin and bone on a human face.

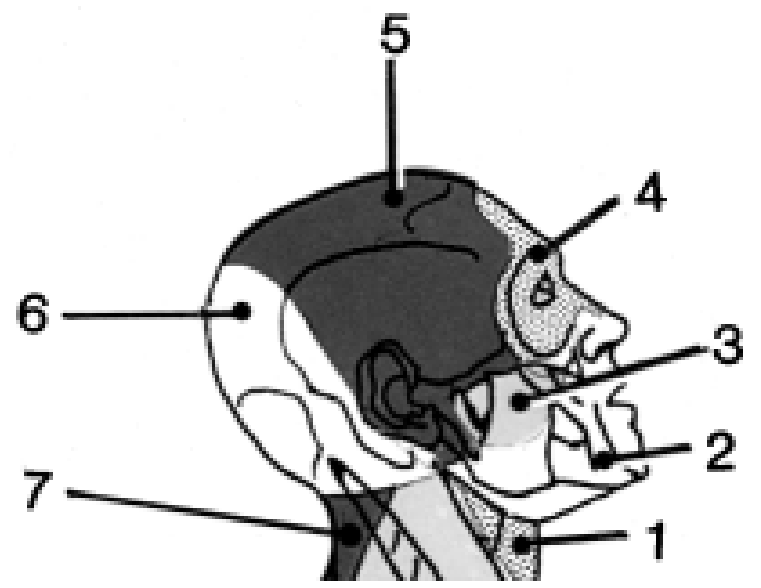

Figure 7. Vascular territories of tissues between skin and bone on face [30].

\subsection{Mapping cardiovascular territories into thermoregulation performance}

In order to understand the behavior of facial cardiovascular territories under different thermal stimuli, we performed a sensitivity analysis between the room temperature (external stimuli) and facial points to investigate how sensitive each territory is to external stimuli. We calculated the statistics (mean $\left(\bar{\mu}_{j}\right.$ (Equation 2) and $\bar{\sigma}_{j}$ (Equation 3)) and standard deviation $\left(\sigma_{\bar{\mu}_{j}}\right.$ (Equation 4) and $\sigma_{\bar{\sigma}_{j}}$ (Equation 5)) of each individual's facial points' statistics (mean $\left(\mu_{j}^{i}\right)$ and standard deviation $\left.\left(\sigma_{j}^{i}\right)\right)$ as shown below.

$\bar{\mu}_{j}=\frac{\sum_{i=1}^{N} \mu_{j}^{i}}{N}$

Equation 2 
$\bar{\sigma}_{j}=\frac{\sum_{i=1}^{N} \sigma_{j}^{i}}{N}$

Equation 3

$\sigma_{\bar{\mu}_{j}}=\sqrt{\frac{\sum_{i=1}^{N}\left(\mu_{j}^{i}-\bar{\mu}_{j}\right)}{N-1}}$

Equation 4

$\sigma_{\bar{\sigma}_{j}}=\sqrt{\frac{\sum_{i=1}^{N}\left(\sigma_{j}^{i}-\bar{\sigma}_{j}\right)}{N-1}}$

Equation 5

Where $\mathrm{N}$ is the number of participants.

Through the analysis of the results, we assessed the individuals' vascular territory behaviors and studied how they are related to thermal comfort.

Table 2. Facial points' statistics in the comfort days

\begin{tabular}{|c|c|c|c|c|c|c|}
\cline { 2 - 6 } \multicolumn{1}{c|}{} & \multicolumn{2}{c|}{ All Participants } & \multicolumn{2}{c|}{ Males } & \multicolumn{2}{c|}{ Females } \\
\cline { 2 - 6 } \multicolumn{1}{c|}{} & $\bar{\mu}_{j} \pm \sigma_{\bar{\mu}_{j}}$ & $\bar{\sigma}_{j} \pm \sigma_{\bar{\sigma}_{j}}$ & $\bar{\mu}_{j} \pm \sigma_{\bar{\mu}_{j}}$ & $\bar{\sigma}_{j} \pm \sigma_{\bar{\sigma}_{j}}$ & $\bar{\mu}_{j} \pm \sigma_{\bar{\mu}_{j}}$ & $\bar{\sigma}_{j} \pm \sigma_{\bar{\sigma}_{j}}$ \\
\hline Cheekbone & $34.41 \pm 0.55$ & $0.58 \pm 0.23$ & $34.70 \pm 0.38$ & $0.51 \pm 0.18$ & $33.90 \pm 0.44$ & $0.71 \pm 0.29$ \\
\hline Ear & $31.79 \pm 1.53$ & $1.43 \pm 0.62$ & $32.12 \pm 1.58$ & $1.63 \pm 0.59$ & $31.23 \pm 1.01$ & $1.07 \pm 0.21$ \\
\hline Front Face & $34.79 \pm 0.46$ & $0.43 \pm 0.16$ & $34.85 \pm 0.48$ & $0.40 \pm 0.19$ & $34.70 \pm 0.47$ & $0.52 \pm 0.06$ \\
\hline Nose & $33.88 \pm 1.03$ & $1.29 \pm 0.54$ & $34.35 \pm 0.75$ & $1.02 \pm 0.5$ & $33.03 \pm 0.99$ & $1.79 \pm 0.08$ \\
\hline Room Temperature & $24.14 \pm 0.48$ & $1.11 \pm 0.33$ & $24.16 \pm 0.51$ & $1.13 \pm 0.4$ & $24.12 \pm 0.48$ & $1.07 \pm 0.28$ \\
\hline Average Facial Points & $33.72 \pm 0.62$ & $0.76 \pm 0.25$ & $34.00 \pm 0.55$ & $0.75 \pm 0.3$ & $33.21 \pm 0.35$ & $0.79 \pm 0.11$ \\
\hline
\end{tabular}

Table 3. Facial points' statistics during cold stress in the extreme day

\begin{tabular}{|c|c|c|c|c|c|c|}
\cline { 2 - 6 } \multicolumn{1}{c|}{} & \multicolumn{2}{|c|}{ All Participants } & \multicolumn{2}{c|}{ Males } & \multicolumn{2}{c|}{ Females } \\
\cline { 2 - 6 } & $\bar{\mu}_{j} \pm \sigma_{\bar{\mu}_{j}}$ & $\bar{\sigma}_{j} \pm \sigma_{\bar{\sigma}_{j}}$ & $\bar{\mu}_{j} \pm \sigma_{\bar{\mu}_{j}}$ & $\bar{\sigma}_{j} \pm \sigma_{\bar{\sigma}_{j}}$ & $\bar{\mu}_{j} \pm \sigma_{\bar{\mu}_{j}}$ & $\bar{\sigma}_{j} \pm \sigma_{\bar{\sigma}_{j}}$ \\
\hline Cheekbone & $33.27 \pm 1.18$ & $0.73 \pm 0.20$ & $33.59 \pm 1.01$ & $0.72 \pm 0.23$ & $32.68 \pm 1.36$ & $0.74 \pm 0.16$ \\
\hline Ear & $30.07 \pm 1.99$ & $1.65 \pm 0.63$ & $30.27 \pm 2.41$ & $1.71 \pm 0.63$ & $29.71 \pm 1.00$ & $1.52 \pm 0.70$ \\
\hline Front Face & $34.06 \pm 0.58$ & $0.47 \pm 0.28$ & $34.14 \pm 0.56$ & $0.49 \pm 0.31$ & $33.93 \pm 0.67$ & $0.43 \pm 0.24$ \\
\hline Nose & $31.70 \pm 2.33$ & $1.73 \pm 0.98$ & $32.73 \pm 1.20$ & $1.73 \pm 0.98$ & $29.83 \pm 2.83$ & $1.73 \pm 1.11$ \\
\hline Room Temperature & $22.32 \pm 1.92$ & $1.91 \pm 0.58$ & $22.70 \pm 2.24$ & $1.90 \pm 0.63$ & $21.64 \pm 1.04$ & $1.95 \pm 0.56$ \\
\hline Average Facial Points & $32.28 \pm 1.09$ & $1.05 \pm 0.46$ & $32.68 \pm 1.02$ & $1.10 \pm 0.44$ & $31.54 \pm 0.87$ & $0.95 \pm 0.55$ \\
\hline
\end{tabular}

Table 4. Facial points' statistics during heat stress in the extreme day 


\begin{tabular}{|c|c|c|c|c|c|c|}
\cline { 2 - 7 } \multicolumn{1}{c|}{} & \multicolumn{2}{c|}{ All Participants } & \multicolumn{2}{c|}{ Males } & \multicolumn{2}{c|}{ Females } \\
\cline { 2 - 7 } & $\bar{\mu}_{j} \pm \sigma_{\bar{\mu}_{j}}$ & $\bar{\sigma}_{j} \pm \sigma_{\bar{\sigma}_{j}}$ & $\bar{\mu}_{j} \pm \sigma_{\bar{\mu}_{j}}$ & $\bar{\sigma}_{j} \pm \sigma_{\bar{\sigma}_{j}}$ & $\bar{\mu}_{j} \pm \sigma_{\bar{\mu}_{j}}$ & $\bar{\sigma}_{j} \pm \sigma_{\bar{\sigma}_{j}}$ \\
\hline Cheekbone & $35.31 \pm 0.71$ & $0.65 \pm 0.55$ & $35.48 \pm 0.77$ & $0.53 \pm 0.46$ & $35.01 \pm 0.51$ & $0.86 \pm 0.69$ \\
\hline Ear & $33.15 \pm 1.76$ & $1.54 \pm 0.68$ & $33.66 \pm 2.00$ & $1.58 \pm 0.85$ & $32.24 \pm 0.69$ & $1.45 \pm 0.21$ \\
\hline Front Face & $35.53 \pm 0.58$ & $0.43 \pm 0.23$ & $35.52 \pm 0.65$ & $0.37 \pm 0.22$ & $35.58 \pm 0.5$ & $0.53 \pm 0.22$ \\
\hline Nose & $34.78 \pm 1.66$ & $0.96 \pm 0.89$ & $35.24 \pm 1.29$ & $0.69 \pm 0.66$ & $33.94 \pm 2.06$ & $1.45 \pm 1.12$ \\
\hline Room Temperature & $26.89 \pm 1.90$ & $1.76 \pm 0.85$ & $27.32 \pm 2.21$ & $1.54 \pm 0.94$ & $26.12 \pm 0.91$ & $2.15 \pm 0.56$ \\
\hline Average Facial Points & $34.70 \pm 1.02$ & $0.85 \pm 0.50$ & $34.98 \pm 1.11$ & $0.74 \pm 0.50$ & $34.19 \pm 0.62$ & $1.05 \pm 0.5$ \\
\hline
\end{tabular}

During the comfort days (Table 2), males had a relatively fixed and close cheekbone and front face temperature behavior (34.70 \pm 0.38 and $34.85 \pm 0.48{ }^{\circ} \mathrm{C}$, respectively), while the nose had a relatively lower temperature $\left(34.35 \pm 0.75{ }^{\circ} \mathrm{C}\right)$ and with higher temperature variations $\left(1.02 \pm 0.5^{\circ} \mathrm{C}\right)$ compared to cheekbone and front face $\left(0.51 \pm 0.18\right.$ and $\left.0.40 \pm 0.19{ }^{\circ} \mathrm{C}\right)$. In males, the ear holds a temperature around $2{ }^{\circ} \mathrm{C}$ lower than other facial points with large variations $\left(1.63 \pm 0.59{ }^{\circ} \mathrm{C}\right)$. Females' facial point measurements were all lower than the similar points in males with similar variations $\left(\bar{\sigma}_{j} \pm\right.$ $\sigma_{\bar{\sigma}_{j}}$ ). Females had a similar behavior in the front face $(0.52 \pm 0.06)$. However, compared to the males, the cheekbone measurements for females were relatively cooler than the front face measurements (about 0.8 $\left.{ }^{\circ} \mathrm{C}\right)$.

Under the cold stress (Table 3), both males and females hold steady temperatures in the front face and cheekbone and their variations are similar to the normal conditions (cheekbone: $0.72 \pm 0.23{ }^{\circ} \mathrm{C}$ for males and $0.74 \pm 0.16{ }^{\circ} \mathrm{C}$ for females, and front face: $0.49 \pm 0.31{ }^{\circ} \mathrm{C}$ for males, $0.43 \pm 0.24{ }^{\circ} \mathrm{C}$ for females). Larger variations were observed in both ear and nose in both genders (ear: $1.71 \pm 0.63{ }^{\circ} \mathrm{C}$ for males and $1.52 \pm 0.70{ }^{\circ} \mathrm{C}$ for females, nose: $1.73 \pm 0.98{ }^{\circ} \mathrm{C}$ for males, $1.73 \pm 1.11{ }^{\circ} \mathrm{C}$ for females). Overall, the drop of temperature on the nose and ears were relatively higher than the drop on the cheekbone and front face. Nose is slightly more sensitive than ears. An important observation is the females held a relatively lower temperature in their nose $\left(\sim 3^{\circ} \mathrm{C}\right)$ than the males.

Under the heat stress (Table 4), the nose, cheekbone, and front face temperatures increased monotonically until they converged to a threshold temperature (a temperature around $36{ }^{\circ} \mathrm{C}$, which is slightly below the normal core temperature), while the ear temperature increased to the values higher than that threshold (see also Figure 6). Ear has the highest variations in both genders $\left(1.58 \pm 0.85^{\circ} \mathrm{C}\right.$ for males and $1.45 \pm 0.21{ }^{\circ} \mathrm{C}$ for females), while the nose was sensitive in females only $\left(0.69 \pm 0.66{ }^{\circ} \mathrm{C}\right.$ for males and $1.45 \pm 1.12{ }^{\circ} \mathrm{C}$ for females). Based on the correlation analysis in the heat stress and cold stress data, while the average points showed high variations $\left(33.72 \pm 0.62{ }^{\circ} \mathrm{C}\right.$ in comfortable days to $32.28 \pm 1.09{ }^{\circ} \mathrm{C}$ under cold stress) when room temperature changed from $24.14 \pm 0.48$ to $22.32 \pm 1.92{ }^{\circ} \mathrm{C}$, the average points did not show high variations in the case of heat stress $\left(33.72 \pm 0.62\right.$ to $34.70 \pm 1.02{ }^{\circ} \mathrm{C}$ when room temperature changed from $24.14 \pm 0.48$ to $\left.26.89 \pm 1.90{ }^{\circ} \mathrm{C}\right)\left(1.44^{\circ} \mathrm{C}\right.$ change in cold stress as opposed to $0.98^{\circ} \mathrm{C}$ in heat stress).

As stated, the cheekbone and the front face had small variations both for each individual and also uniform across individuals under different environmental conditions (normal conditions: $0.73 \pm 0.20{ }^{\circ} \mathrm{C}$ for the cheekbone and $0.47 \pm 0.28{ }^{\circ} \mathrm{C}$ for the front face, cold stress: $0.73 \pm 0.20{ }^{\circ} \mathrm{C}$ for the cheekbone and $0.47 \pm 0.28{ }^{\circ} \mathrm{C}$ for the front face, heat stress: $0.65 \pm 0.55{ }^{\circ} \mathrm{C}$ for the cheekbone and $0.43 \pm 0.23{ }^{\circ} \mathrm{C}$ for the 
front face). However, both the ear and nose had relatively large variations for each participant with a uniform behavior across all participants (normal conditions: $1.43 \pm 0.62{ }^{\circ} \mathrm{C}$ for the ear and $1.29 \pm 0.54{ }^{\circ} \mathrm{C}$ for the nose, cold stress: $1.65 \pm 0.63{ }^{\circ} \mathrm{C}$ for the ear and $1.73 \pm 0.98{ }^{\circ} \mathrm{C}$ for the nose, heat stress: $1.54 \pm$ $0.68{ }^{\circ} \mathrm{C}$ for the ear and $0.96 \pm 0.89^{\circ} \mathrm{C}$ for the nose).

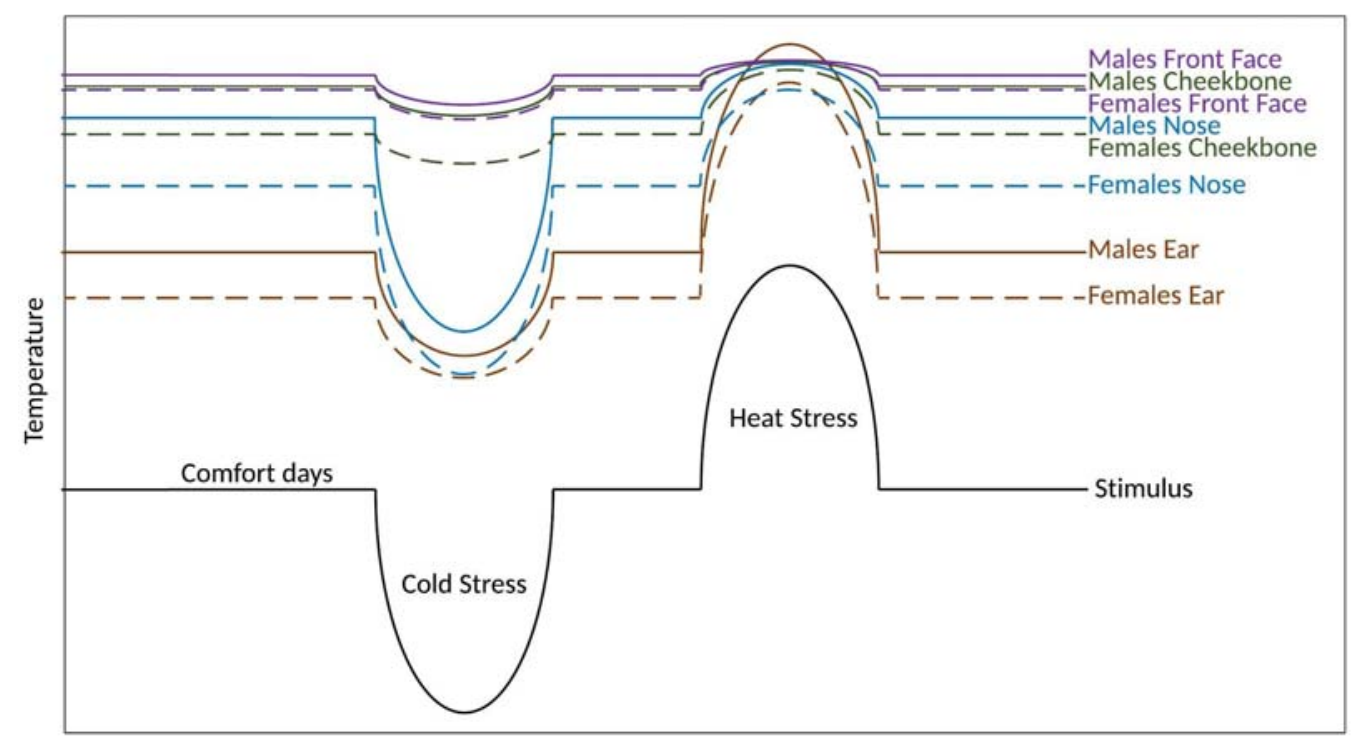

Figure 8. Illustration of the observed physiological behavior for males, females and combined population

Figure 8 illustrates the typical response observed across participants based on the variations (quantified based on the standard deviation measure) during the heat and cold stresses in a schematic fashion. The vertical axis does not have a unit due to the fact that the absolute values of temperatures were slightly different from participant to participant. In response to the cold stress, the temperatures of the front face and cheekbone slightly dropped, however the temperatures of ear and nose dropped relatively higher. The nose showed a greater response during the cold stress compared to the ear. In response to the heat stress, the front face and cheekbone gained a relatively small increase in temperature, while the nose had relatively larger increase. All of the three points converge to a temperature threshold eventually. On the other hand, the ear's temperature showed a continuous growth, exceeded the other points' temperature and approached the core body temperature $\left(\sim 37^{\circ} \mathrm{C}\right)$. The figure also demonstrates the gender driven variations in responses and suggests that although females had relatively lower skin temperature on all of the points, they experienced the neutral environment colder compared to males.

\subsection{Mapping thermoregulation performance into personal thermal comfort}

Based on the observed physiological behaviors (Figure 8) and subjective thermal votes, we defined two heuristics for determining thermally uncomfortable conditions. As the temperatures of the nose and ear go below the temperature of the cheekbone and the front face, the probability of thermally uncomfortably cool conditions increases. We define $\Delta_{1}$ as the average temperature of nose and ear, minus the average temperature of front face and cheekbone. As the temperature of the ear approaches the temperature of the cheekbone, front face, and nose, the probability of uncomfortably warm conditions increases. We define a $\Delta_{2}$ as the difference between the temperature of the ear and the average temperature of the cheekbone, 
front face, and nose. In order to check if there is any similarity between the participants in terms of $\Delta_{1}$ and $\Delta_{2}$, we matched the thermal comfort votes with instant temperature measurements of each individual's facial points at the time of voting (using the UI shown in Figure 4). We then calculated the $\Delta_{1}$ for the uncomfortably cool votes, and $\Delta_{2}$ for the uncomfortably warm votes for each individual for both female and male participants. In order to visualize how $\Delta_{1}$ and $\Delta_{2}$ differ based on gender, we calculated the percentiles as a function of their values. Percentiles indicate the value below which a given percentage of observations in a group of observations fall. It is calculated based on a relatively simple formula (Equation 6).

$\mathrm{P}(\mathrm{x})=\frac{\mathrm{n}(\mathrm{x})}{\mathrm{N}}$

Equation 6

Where $\mathrm{P}(\mathrm{x})$ is the percentile of the observed value, $\mathrm{n}(\mathrm{x})$ is the rank of observed value in an ordered (e.g., decreasing or increasing) list, $\mathrm{N}$ is the total number of observations.

The data collected from 15 participants included 115 uncomfortable votes and 469 comfortable votes (total 584 votes). Out of the 115 uncomfortable votes, 45 votes were for uncomfortably cool and 70 votes were for uncomfortably warm. We focused on defining the heuristics and confidence intervals for the uncomfortable conditions because predicting and addressing the uncomfortable conditions is the main goal of a learning system. Figures 9 and 10 demonstrate the uncomfortably cool and warm thermal votes $\left(\Delta_{1}\right)$ percentiles, respectively. As it can be seen in Figure 10, there is a gap between the female and male perceptions of uncomfortably warm conditions (females' thermoregulation system responses are less sensitive to the perception of warm conditions). This finding also demonstrates that the perception of warm conditions is associated with a larger response in the thermoregulation. On the other hand, there is not a considerable gap between the females' and males' percentiles for the uncomfortably warm conditions (Figure 9), which suggests that variation in the perceptions of the thermal environment for cool conditions across genders are small. We then took the 95 percentile confidence interval of $\Delta_{1}$ and $\Delta_{2}$ across female and male participants. The 95 percentile confidence for $\Delta_{1}$ and $\Delta_{2}$ are $-5.9{ }^{\circ} \mathrm{C}$ and $-0.9{ }^{\circ} \mathrm{C}$ for females and $-5.3{ }^{\circ} \mathrm{C}$ and $1.1{ }^{\circ} \mathrm{C}$ for males, respectively. The calculations for both the mean and confidence interval of $95 \%$ for $\Delta_{1}$ and $\Delta_{2}$ suggest that the $\Delta_{1}$ and $\Delta_{2}$ can be used to describe the thermoneutral zone, as well as to estimate thermal comfort. For example, once $\Delta_{1}$ goes below $-5.9{ }^{\circ} \mathrm{C}$ for females, it is very likely (with more than $95 \%$ ) that they perceive the environment as uncomfortably cool. Similarly, if $\Delta_{2}$ gets to values greater than $-0.9{ }^{\circ} \mathrm{C}$ it is very likely (with more than $95 \%$ ) that they perceive the environment as uncomfortably warm. In addition, when $\Delta_{1}$ and $\Delta_{2}$ metrics start to grow toward the values that increase confidence levels for uncomfortably warm and cool conditions, it implies that the participant's thermoregulation system is moving away from a thermoneutral zone. The generation of $\Delta_{1}$ and $\Delta_{2}$ across genders and forming confidence levels that capture thermally uncomfortable conditions for all participants in our experiment suggests that thermal discomfort signatures can potentially be generalized across population, which can result in a considerable reduction and eventually elimination of the training of comfort prediction algorithms. 


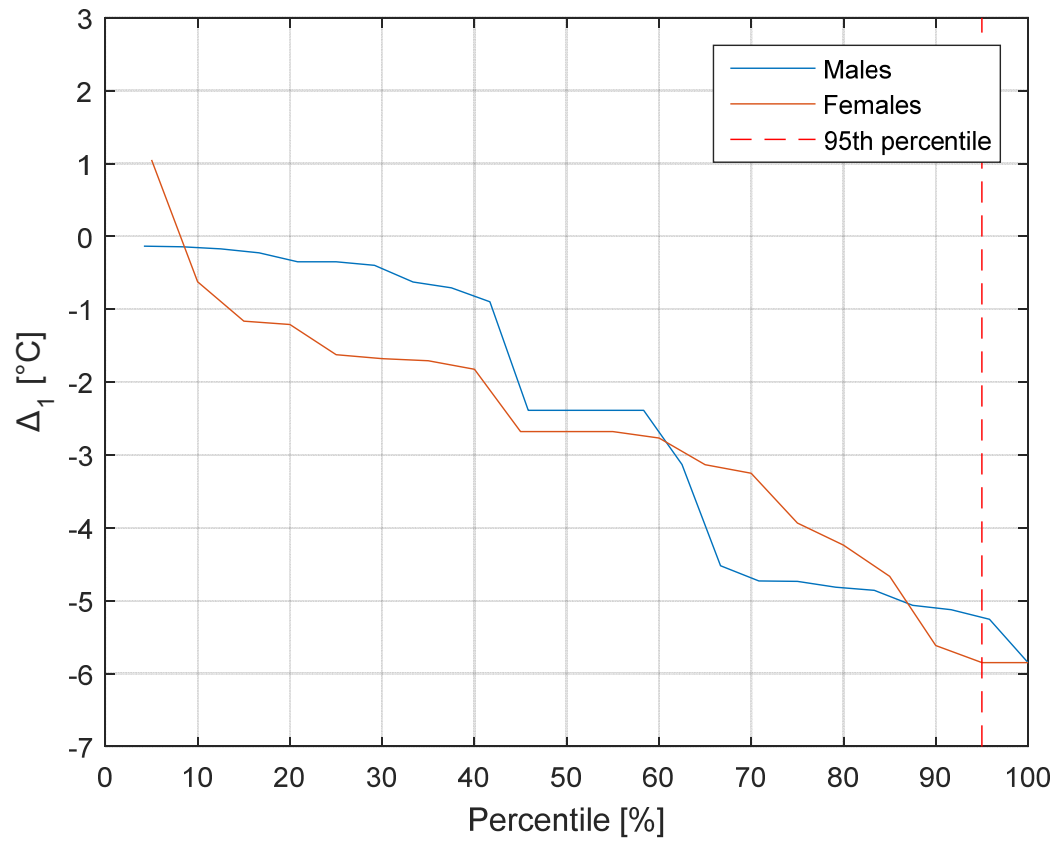

Figure 9. Uncomfortably cool conditions metric $\left(\Delta_{1}\right)$ across all participants' votes

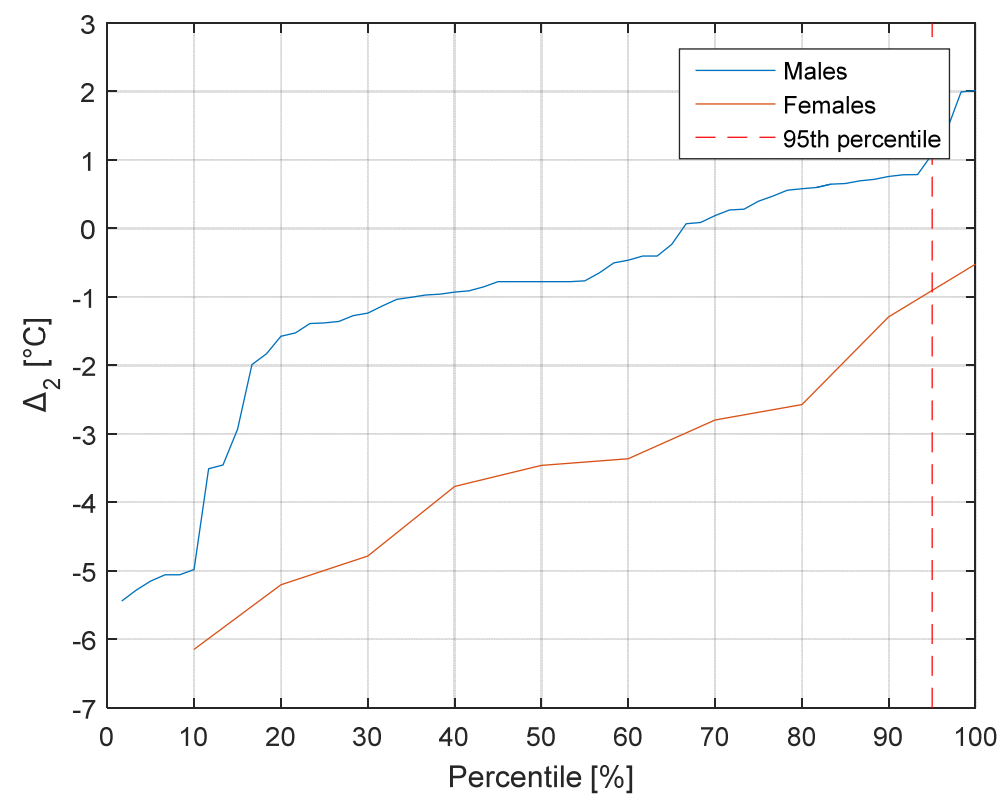

Figure 10. Uncomfortably warm conditions metric $\left(\Delta_{2}\right)$ across all participants' votes

\section{DISCUSSION}

Real-time monitoring of the facial skin blood flow enabled us to study the human thermoregulatory performance under different thermal stimulus in an office work environment. Our results demonstrate that although vasodilation and vasoconstriction are the driving factors in the facial temperature variations, the monitored facial points' behaviors are not similar except for the front face and cheekbone. Such 
phenomena can be described based on the underlying vascular territories, and it explains the facial points' variant behaviors under the heat and cold stresses. Through defining heuristics based on the observed thermoregulation performance, we calculated confidence intervals for predicting thermally uncomfortable conditions for males and females. Our results demonstrated that there is a gap between thermally uncomfortable conditions in females and males. The presented results are very promising, however, there are some limitations, which we plan to address in our future studies. For example, the impact of other influential factors on skin blood flow, such as activity level or any other physiological factors were minimal and not considered in the analysis. These factors may influence the blood flow and thus skin temperature as stated in Section 3. In addition, the data collection was performed during relatively warmer climate conditions and consequently, the impact of climatic variations was not considered in our study. In this study, we used infrared measurements for the vasodilation and vasoconstriction mechanisms to monitor thermoregulation performance. Other physiological responses to thermal stresses, such as sweating might impact the infrared measurements, as the skin surface characteristics may change. However, infrared measurements would reflect the thermoregulation performance as it is trying to release the heat from body during a heat stress before the sweating begins. Due to the temperature ranges we using during the heating stress period of our experiments, none of the participants reported any sweating during the data collection. The validation results were driven based on the data collected for 15 participants, thus further exploration with larger sample size is required to generalize the observed behavior across the population.

The prediction of thermal comfort via our proposed non-intrusive and contact-less technique enables smart service systems, such as energy efficient buildings that are also responsive to the occupants. In a previous study, we demonstrated that given the real-time personal comfort information is available, personal thermal comfort requirements can be integrated into the HVAC system's control logic for multi occupancy spaces by defining a set of operational constraints [12]. The presented technique in this paper has the capability to provide the required comfort information in real time. Defining the thermal comfort requirements as a set of constraints transforms the legacy HVAC systems' multi-objective optimization (with objectives of maximizing comfort and minimizing energy consumption) to solely one objective, which is energy efficiency (constrained by thermal comfort requirements). Such modeling prevents Pareto optimality problems related to a multi-objective optimization. In other words, building systems' operations energy costs can be optimized based on the control parameters (i.e., setpoint and deadband) subject to the constraints of personal thermal comfort requirements and other indoor environmental quality requirements. This allows to further improve the energy efficiency in buildings. The potential energy savings from optimizing the building HVAC temperature setpoints can reach up to 37\% [14] depending on the climate, building size, and materials. While the more sophisticated optimization and control algorithms might help improving the energy efficiency and thermal comfort, there is a trade-off between the controller complexity and the potential energy savings [45-48], which requires further investigations. The benefits of the control policy mentioned above for HVAC operations is not limited to energy efficiency. For example, it has been shown that workplace productivity can be improved when occupants have the control over their thermal environment and their thermal comfort has been fulfilled [49-52].

The proposed sensing technique can also have other applications where physiological processes may impact skin blood flow. For example, the technique could be used in monitoring thermal stress in workers, who work in extreme thermal conditions and face hyperthermia and hypothermia in outdoor 
environments (e.g., construction workers). Regions with hot climates experience periods of very high temperatures [53] with an increase in the frequency and intensity of heat waves due to the climate change, which has increased the number of fatalities and illnesses caused by hot weather [54]. Another application area of the sensing technique relates to the internal medicine. During menopause, changes in reproductive hormone levels substantially alter thermoregulatory control of skin blood flow. In type 2 diabetes mellitus, the ability of skin blood vessels to dilate is impaired. This impaired vasodilation in both cases likely contributes to the increased risk of illnesses during exposure to elevated ambient temperatures. Further research is required to investigate these new applications of infrared thermography for indirect skin blood flow measurements.

\section{CONCLUSIONS}

In this paper, we presented a novel infrared thermography based technique to monitor the skin blood flow variations measured indirectly through the skin temperature on several points on human face. We selected human face because it has a high density of blood vessels and it is usually not covered by clothing. During the four days of data collection, participants were asked to communicate their comfort feedback while performing regular office activities. Our sensing system consisted of an eyeglass equipped with four infrared sensors to collect infrared radiations on four points on human face (i.e., front face, cheekbone, nose, and ear) and four temperature/humidity sensors located around the participants to monitor environmental conditions and thermal stimuli. We quantitatively studied the thermoregulatory performance, namely vasodilation and vasoconstriction, via the variations in the skin temperature under external thermal stimuli. We demonstrated how thermoregulatory performance, the behaviors of the vascular territories, can be used to estimate personal thermal comfort levels. We defined two heuristics for detecting cold and hot conditions at the individual level and searched for generalizing it across individuals. Our results show that the proposed heuristics for both uncomfortably cool and uncomfortably warm conditions can provide confidence levels of up to $95 \%$ for comfort prediction. In addition, considerable variations were observed in the thermoregulation performance and uncomfortably warm conditions metrics between the males and females. For example, females' thermoregulation system responses are less sensitive to the perception of the warm conditions. A similar behavior was observed for uncomfortably cool conditions across genders. Our proposed technique allows for continuous monitoring of thermoregulation performance, as well as instantaneous identification of thermal comfort during daily office activities. The information learned based on our proposed method can be used as constraints for the optimization of HVAC system operations in buildings and considerable HVAC related energy savings and increase in occupant satisfaction could potentially be achieved by selecting optimal control parameters.

\section{ACKNOWLEDGMENTS}

This material is based upon work supported by the National Science Foundation under Grant No. 1351701. Any opinions, findings, and conclusions or recommendations expressed in this material are those of the authors and do not necessarily reflect the views of the National Science Foundation.

\section{REFERENCES}

[1] U. EIA, Annual energy outlook 2013, US Energy Information Administration, Washington, DC (2013). 
[2] C. Initiative, Buildings and Climate Change (2009).

[3] L. Pérez-Lombard, J. Ortiz, C. Pout, A review on buildings energy consumption information, Energy and Buildings. 40 (2008) 394-398.

[4] A. Standard, Standard 55-2004, Thermal environmental conditions for human occupancy (2004).

[5] A. Standard, Standard 62.1-2010 (2010). Ventilation for Acceptable Indoor Air Quality, Atlanta, GA, American Society of Heating, Refrigerating and Air-Conditioning Engineers, Inc.

[6] G. Jendritzky, R. de Dear, Adaptation and thermal environment, in: Biometeorology for adaptation to climate variability and change, Springer, 2009, pp. 9-32.

[7] G.S. Brager, R.J. de Dear, Thermal adaptation in the built environment: a literature review, Energy and Buildings. 27 (1998) 83-96.

[8] W.F. Song, C.J. Zhang, D.D. Lai, F.M. Wang, K. Kuklane, Use of a novel smart heating sleeping bag to improve wearers' local thermal comfort in the feet, Scientific reports. 6 (2016) 19326.

[9] A. Ghahramani, F. Jazizadeh, B. Becerik-Gerber, A knowledge based approach for selecting energyaware and comfort-driven HVAC temperature set points, Energy and Buildings. 85 (2014) 536-548.

[10] J.F. Nicol, M.A. Humphreys, Adaptive thermal comfort and sustainable thermal standards for buildings, Energy and Buildings. 34 (2002) 563-572.

[11] A. Ghahramani, C. Tang, Z. Yang, B. Becerik-Gerber, A Study of Time-Dependent Variations in Personal Thermal Comfort via a Dynamic Bayesian Network, in: Sustainable Human-Building Ecosystems, pp. 99-107.

[12] A. Ghahramani, C. Tang, B. Becerik-Gerber, An online learning approach for quantifying personalized thermal comfort via adaptive stochastic modeling, Building and Environment. 92 (2015) 8696.

[13] A. Ghahramani, K. Dutta, Z. Yang, G. Ozcelik, B. Becerik-Gerber, Quantifying the influence of temperature setpoints, building and system features on energy consumption (2015) 1000-1011.

[14] A. Ghahramani, K. Zhang, K. Dutta, Z. Yang, B. Becerik-Gerber, Energy savings from temperature setpoints and deadband: Quantifying the influence of building and system properties on savings, Applied Energy. 165 (2016) 930-942.

[15] C. Huizenga, H. Zhang, E. Arens, D. Wang, Skin and core temperature response to partial-and whole-body heating and cooling, Journal of thermal biology. 29 (2004) 549-558.

[16] S. Takada, S. Matsumoto, T. Matsushita, Prediction of whole-body thermal sensation in the nonsteady state based on skin temperature, Building and Environment. 68 (2013) 123-133.

[17] H. Liu, J. Liao, D. Yang, X. Du, P. Hu, Y. Yang, B. Li, The response of human thermal perception and skin temperature to step-change transient thermal environments, Building and Environment. 73 (2014) 232-238. 
[18] H. Hensel, Thermoreception and temperature regulation, Monographs of the Physiological Society. 38 (1981) 1-321.

[19] A.A. Romanovsky, Thermoregulation: some concepts have changed. Functional architecture of the thermoregulatory system, American journal of physiology.Regulatory, integrative and comparative physiology. 292 (2007) R37-46.

[20] A. Auliciems, S.V. Szokolay, Thermal comfort (1997).

[21] K.C. Bicego, R.C. Barros, L.G. Branco, Physiology of temperature regulation: comparative aspects, Comparative Biochemistry and Physiology Part A: Molecular \& Integrative Physiology. 147 (2007) 616639.

[22] A. Simone, J. Kolarik, T. Iwamatsu, H. Asada, M. Dovjak, L. Schellen, M. Shukuya, B.W. Olesen, A relation between calculated human body exergy consumption rate and subjectively assessed thermal sensation, Energy and Buildings. 43 (2011) 1-9.

[23] Q. Zhao, Y. Zhao, F. Wang, J. Wang, Y. Jiang, F. Zhang, A data-driven method to describe the personalized dynamic thermal comfort in ordinary office environment: From model to application, Building and Environment. 72 (2014) 309-318.

[24] Y. Yao, Z. Lian, W. Liu, C. Jiang, Y. Liu, H. Lu, Heart rate variation and electroencephalograph-the potential physiological factors for thermal comfort study, Indoor air. 19 (2009) 93-101.

[25] W. Liu, Z. Lian, Q. Deng, Y. Liu, Evaluation of calculation methods of mean skin temperature for use in thermal comfort study, Building and Environment. 46 (2011) 478-488.

[26] S.Y. Sim, M.J. Koh, K.M. Joo, S. Noh, S. Park, Y.H. Kim, K.S. Park, Estimation of Thermal Sensation Based on Wrist Skin Temperatures, Sensors. 16 (2016) 420.

[27] K. Nakayama, T. Suzuki, K. Kameyama, Estimation of thermal sensation using human peripheral skin temperature (2009) 2872-2877.

[28] D. Wang, H. Zhang, E. Arens, C. Huizenga, Observations of upper-extremity skin temperature and corresponding overall-body thermal sensations and comfort, Building and Environment. 42 (2007) 39333943.

[29] B.R. Kingma, A.J. Frijns, L. Schellen, van Marken Lichtenbelt, Wouter D, Beyond the classic thermoneutral zone: including thermal comfort, Temperature. 1 (2014) 142-149.

[30] G.I. Taylor, The blood supply of the skin, Grabb and Smith's Plastic Surgery, 5th Ed.Philadelphia: Lippincott-Raven (1997) 47-59.

[31] L.B. Rowell, Cardiovascular adjustments to thermal stress, Comprehensive Physiology.

[32] J.M. Johnson, G.L. Brengelmann, J.R. Hales, P.M. Vanhoutte, C.B. Wenger, Regulation of the cutaneous circulation, Federation proceedings. 45 (1986) 2841-2850. 
[33] J.M. Johnson, D.W. Proppe, Cardiovascular adjustments to heat stress, Comprehensive Physiology (2011).

[34] N. Charkoudian, Skin blood flow in adult human thermoregulation: how it works, when it does not, and why. 78 (2003) 603-612.

[35] P.E. Pergola, D.L. Kellogg Jr, J.M. Johnson, W.A. Kosiba, Reflex control of active cutaneous vasodilation by skin temperature in humans, The American Journal of Physiology. 266 (1994) H1979-84.

[36] P.E. Pergola, J.M. Johnson, D.L. Kellogg Jr, W.A. Kosiba, Control of skin blood flow by whole body and local skin cooling in exercising humans, The American Journal of Physiology. 270 (1996) H208-15.

[37] J.S. Petrofsky, Resting blood flow in the skin: does it exist, and what is the influence of temperature, aging, and diabetes?, Journal of diabetes science and technology. 6 (2012) 674-685.

[38] I.D. Swain, L.J. Grant, Methods of measuring skin blood flow, Physics in Medicine and Biology. 34 (1989) 151-175.

[39] I.B. Wilkinson, D.J. Webb, Venous occlusion plethysmography in cardiovascular research: methodology and clinical applications, British journal of clinical pharmacology. 52 (2001) 631-646.

[40] Y. Chen, B. Lu, Y. Chen, X. Feng, Breathable and Stretchable Temperature Sensors Inspired by Skin, Scientific reports. 5 (2015).

[41] M.C. Peel, B.L. Finlayson, T.A. McMahon, Updated world map of the Köppen-Geiger climate classification, Hydrology and Earth System Sciences Discussions. 4 (2007) 439-473.

[42] J.A.O. García, A review of general and local thermal comfort models for controlling indoor ambiences, Air Quality.InTech, Rijeka (2010).

[43] M.D. Sheet, MLX90614 family, single and dual zone infrared thermometer in TO-39 (2009).

[44] G.I. Taylor, J. Palmer, The vascular territories (angiosomes) of the body: experimental study and clinical applications, British journal of plastic surgery. 40 (1987) 113-141.

[45] H.N. Rafsanjani, C.R. Ahn, M. Alahmad, A Review of Approaches for Sensing, Understanding, and Improving Occupancy-Related Energy-Use Behaviors in Commercial Buildings, Energies. 8 (2015) 10996-11029.

[46] F. Zhang, R. de Dear, C. Candido, Thermal comfort during temperature cycles induced by direct load control strategies of peak electricity demand management, Building and Environment. 103 (2016) 9-20.

[47] S. Ahmadi-Karvigh, B. Becerik-Gerber, L. Soibelman, A framework for allocating personalized appliance-level disaggregated electricity consumption to daily activities, Energy and Buildings. 111 (2016) 337-350. 
[48] H.N. Rafsanjani, C.R. Ahn, M. Alahmad, Development of Non-Intrusive Occupant Load Monitoring (NIOLM) in Commercial Buildings: Assessing Occupants' Energy-Use Behavior at Entry and Departure Events, in: Sustainable Human-Building Ecosystems, pp. 44-53.

[49] S. Tanabe, M. Haneda, N. Nishihara, Workplace productivity and individual thermal satisfaction, Building and Environment. 91 (2015) 42-50.

[50] P.M. Bluyssen, Towards new methods and ways to create healthy and comfortable buildings, Building and Environment. 45 (2010) 808-818.

[51] D. Heinzerling, S. Schiavon, T. Webster, E. Arens, Indoor environmental quality assessment models: A literature review and a proposed weighting and classification scheme, Building and Environment. 70 (2013) 210-222.

[52] A.C. Boerstra, M. te Kulve, J. Toftum, M.G. Loomans, B.W. Olesen, J.L. Hensen, Comfort and performance impact of personal control over thermal environment in summer: Results from a laboratory study, Building and Environment. 87 (2015) 315-326.

[53] A.J. McMichael, R.E. Woodruff, S. Hales, Climate change and human health: present and future risks, The Lancet. 367 (2006) 859-869.

[54] M.L. Parry, Climate Change 2007: impacts, adaptation and vulnerability: contribution of Working Group II to the fourth assessment report of the Intergovernmental Panel on Climate Change, Cambridge University Press, 2007. 